\title{
Elemental Analysis Using Atomic Absorption Spectroscopy
}

\author{
Abdul Moiz Mohammed
}

\begin{abstract}
The resonance radiation study is a powerful means in obtaining valuable information concerning the behavior of individual atoms and molecules. Various quantitative techniques are available in order to determine the amount of analyte and analysis of trace elements in the sample. Some technique uses the principle, when the number of atoms in the path of light increases, the amount of light absorption increases. While other techniques use dissociation of chemical compound free atoms by supplying enough thermal energy. In this paper, an attempt is made to compare various analytical methods with their merits and demerits and the reasons for popularity of atomic absorption spectroscopy among researchers. Elemental analysis using atomic absorption spectroscopy of various elements that are found in nature particularly in foods and environment is also presented.
\end{abstract}

Keywords - analytical methods, atomic absorption spectroscopy, flame spectroscopy, trace elements.

\section{INTRODUCTION}

In 1850s, Kirchoff and Bunsen used atomic emission along with atomic absorption spectroscopy for the identification of atoms in hot gases and flames. Indeed, it's been a century in the development of analytic investigation employing atomic emission. Moreover, it was during 1950s to late 1960s, when Walsh and Alkemede experimented with industrial equipment which led to the term 'Modern atomic absorption spectroscopy' [1]. However, it was due to an experiment of Walsh with sodium discharge lamp and gas flame (called as flame atomic absorption spectroscopy - Flame AAS), he pointed out the importance of atomic absorption spectra in the spectroscopic studies of molecules; and measurement of emitted radiation in studies of atoms [2]. The development of electro-thermal atomic absorption spectrometry (ET AAS) is credited to the hard work of L'vov for about 10 years, where he experimented with isothermal furnace i.e., graphite cuvette. Later, Massmann's simplified design of an isothermal furnace using additional electrode (electrothermal atomizer) formed the basis for the development of the stabilized temperature platform furnace (STPF) by Slavin in 1978 [3].

During the end of the 20th century, Bernhard Welz considered the prospect of AAS with the development of following three main aspects, which are as follows: a) utilization of semiconductor diode lasers (SDL) as an exciting source; as SDL produce stable energy \& several order of magnitude than that of any conventional source, b) solid samples for ET-AAS; as solid pure metals \& alloys are rigid, heat resistant and difficult to dissolve; and c) continuum source AAS [4].

\section{THEORETICAL ASPECT\& INSTRUMENTATION}

Fraunhofer lines, commonly known as absorption lines in the spectrum of sun were first observed by Wollaston and explained by Brewster in 1832; when the later observed the absorption of white light in an absorbing vapor. Systematic investigation of reversal spectral lines from light exposed $\mathrm{Li}$, $\mathrm{Na}, \mathrm{K}, \mathrm{Ca}$, Sr, and $\mathrm{Br}$ were credited to Liveing, Dewar and Kirchhoff. The principle involved in the flame spectroscopy is to attain unequal equilibrium in temperature of source of light and vapor, which results in formation of lines due to emission or absorption. The vapor introduced in the flame mostly consists of metallic elements that are in an unexcited and unionized ground state. These atoms receive energy from a desired wavelength of radiation, making them to excite to allowed higher energy state. The quantitative analysis such as determination of concentration, examining the molecule property using Beer-Lambert law is mentioned in detail elsewhere [5].

If $E_{1}$ and $E_{2}$ are energy of two states in an assembly of atoms and electrons, then the amount of atoms in these two states are given by:

$$
n_{1}=C g_{1} e^{-\frac{E_{1}}{k T}}
$$

and

$$
n_{2}=C g_{2} e^{-\frac{E_{2}}{k T}}
$$

where, $C$ is a constant independent of $E, T$ is temperature, $g_{1}$ and $g_{2}$ statistical weights of individual state, and $k$ is Boltzmann distribution constant. For total quantum number J, each statistical weight $p$ will have $(2 J+1)$ values, while magnetic quantum number $m_{J}$ has $(2 J+1)$ different values.

The ratio of the number of atoms in excited state to that of number of atoms in ground state is given by:

$$
\frac{n_{2}}{n_{1}}=\frac{g_{2}}{g_{1}} e^{\frac{\Delta E}{k T}}
$$


Here, $\Delta E$ is the energy difference and $E=h v=\frac{h c}{\lambda}$; $c, v$ and $\lambda$ are speed of light, frequency, and wavelength of absorbed radiation respectively.

The distribution of free atoms that absorb at various electronic energy levels may be represented by:

$$
\frac{n_{2} \Delta E}{\tau}=\frac{n_{1} \Delta E g_{2}}{\tau g_{1}} e^{\frac{\Delta E}{k T}}
$$

where $\tau$ is the lifetime in the excited state.

The basic requirement of Beer-Lambert law is homogeneity of sample and requires a linear relation between the concentration and path length. However, population of free atoms in a flame is very low in degree of homogeneity. The absorption for each element in the flame and absorption line depends on the strength of oscillator which can be given by:

$$
\int_{0}^{\infty} K_{v} d_{v}=\frac{\pi e^{2}}{m c} N f
$$

where $e$ and $m$ is the charge and mass of electron, $N$ is the number of free atoms absorbing the incident light and $f$ is the oscillator strength of the absorption line [6].

It is to note that the atomic absorption which is a physical property of an element is independent of temperature, while production of free atoms in a flame is dependent on temperature [7].

The most important components of an atomic absorption spectrometer are shown in the figure (1). The components are: a) light source, which emits a narrow line spectrum of the element of interest, b) absorption cell, also called atom reservoir in which atoms of interest are dissociated by thermal (flame) treatment, c) monochromator used for isolation of wavelength, d) photomultiplier detector, which will convert photons of light into an electrical signal, and e) amplifier and computer [8].

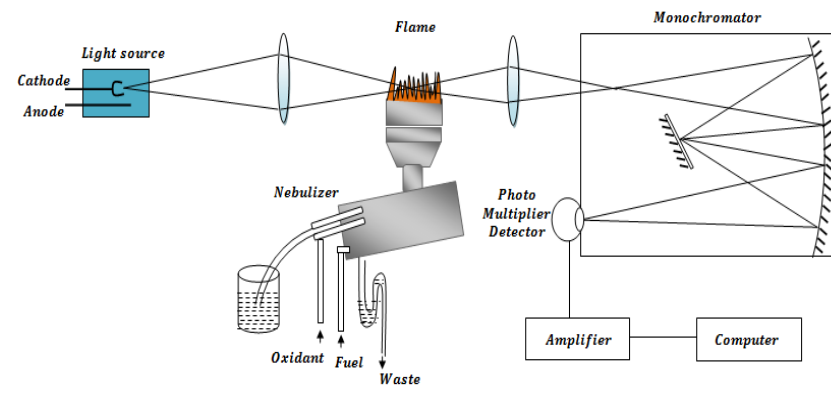

Fig. 1. Block diagram of a basic Atomic Absorption Spectrometer.

A hollow cathode lamp filled with deuterium or hydrogen is used as continuum or white light source, having an emission spectrum in the UV range of the spectrum. An aerosol made up droplets of different sizes are produced by passing the sample solution using the nebulizer through a capillary tube. An adjustable nebulizer is employed to optimizes the uptake rate of the sample solution depending on viscosity and surface tension. The most common flame use in atomizer is shown in Table I.
TABLE I: CHARACTERISTIC OF MOST COMMON FLAME USED IN ATOMIZER

\begin{tabular}{cccc}
\hline \hline Fuel & Oxidant & $\begin{array}{c}\text { Approximate } \\
\text { Temperature }\left({ }^{\circ} \mathrm{C}\right)\end{array}$ & $\begin{array}{c}\text { Burning Velocity } \\
\left(\mathrm{ms}^{-1}\right)\end{array}$ \\
\hline Acetylene & Air & $2100-2300$ & $1.58-2.66$ \\
Acetylene & $\begin{array}{c}\text { Nitrous } \\
\text { Oxide }\end{array}$ & $2600-2950$ & 2.85 \\
\hline
\end{tabular}

TABLE II: INSTRUMENT ANALYSIS CONDITION IN ATOMIC

\begin{tabular}{|c|c|c|c|c|c|}
\hline \multirow[t]{2}{*}{ Metal } & \multirow{2}{*}{$\begin{array}{l}\text { Wavelength } \\
(\mathrm{nm})\end{array}$} & \multirow[t]{2}{*}{ Slit $(n m)$} & \multirow{2}{*}{$\begin{array}{c}\text { Lamp } \\
\text { Current } \\
(m A)\end{array}$} & \multicolumn{2}{|c|}{$\begin{array}{c}\text { Gas Flow } \\
\left(L \cdot \min ^{-1}\right)\end{array}$} \\
\hline & & & & Acetylene & Air \\
\hline$Z n$ & 213.9 & 0.7 & 10 & 2.4 & 10 \\
\hline $\mathrm{Pb}$ & 217.0 & 0.2 & 10 & 2.5 & 10 \\
\hline$N i$ & 232.0 & 0.1 & 15 & 2.6 & 10 \\
\hline$F e$ & 248.3 & 0.2 & 40 & 2.5 & 10 \\
\hline$M n$ & 279.5 & 0.2 & 10 & 2.8 & 10 \\
\hline$M g$ & 285.2 & 0.4 & - & 2.2 & 10 \\
\hline $\mathrm{Cu}$ & 324.7 & 0.7 & 12 & 2.6 & 10 \\
\hline $\mathrm{Ca}$ & 422.7 & 0.7 & 10 & 3.5 & 10 \\
\hline$K$ & 766.5 & 0.4 & - & 2.2 & 10 \\
\hline
\end{tabular}
ABSORPTION SPECTROSCOPY

Monochromator isolates the resonance line by excluding all other wavelengths reaching the detector. A diffraction grating monochromator is universally used in atomic absorption devices. A photomultiplier tube will convert the radiant energy into electrical signal for amplification and data analysis by the computer program [8].

\section{COMPARISON OF ANALYTICAL METHODS}

There are various analytical methods for the identification of elements such as Potentiometry, Voltammetry, Atomic spectroscopy, X-ray techniques and Nuclear techniques. In the following section, a brief comparison of these methods is presented, which will help in selecting the suitable method depending on the specification of the sample or the element which is to be analyzed.

Potentiometry, it is an electro-analytical method used to study an analyte by measuring potential of a solution between two electrodes or by measuring current in an electrochemical cell that contains the analyte. This method directly measures free ions or elements present in an analyte. The possible interferences that occur in this method is due to completing ions in the sample and has a detection limit $10^{-8}$ to $10^{-11}$. However, this method is very sensitive to ionic strength, $\mathrm{pH}$ and requires frequent calibration. On the other hand, Voltammetry is a three electrode system that measures electrochemical activity of an analyte or measures the current in an analyte under variable or constant potential difference. It requires a small amount of sample in element or free ion state and can measure mass fraction in the range of $10^{-10}$ to $10^{-12}$ limit. The drawback of this method is its repeatability, limited accuracy in measuring residual current of analyte.

$\mathrm{X}$-ray scattering method is a non-destructive analytical method used to investigate crystal structure, physical properties of a material and understanding chemical composition by measuring the scattered intensity of X-ray beam from the sample. The prominent feature of this method is investigation of total element concentration in the same range of voltammetry. The limitations of this method are nonideal behavior and difficulty in calibrating the operating 
instrument. Nuclear analytical techniques are employed for the validation of elements in various fields such as environmental and material science, medicine, geology, and cosmology. These techniques may be subdivided into many groups such as measurement of radionuclides, nuclear magnetic resonance and Mosbauer spectroscopy. An analyte in this method can be taken in any of ion/element form or labile complexes, where it can measure total elemental concentration in the detection range of $10^{-12}$ to $10^{-15}$. The main drawback of these techniques is its low throughput, nonideal behavior, and high cost of measuring equipment and preparation of the sample.
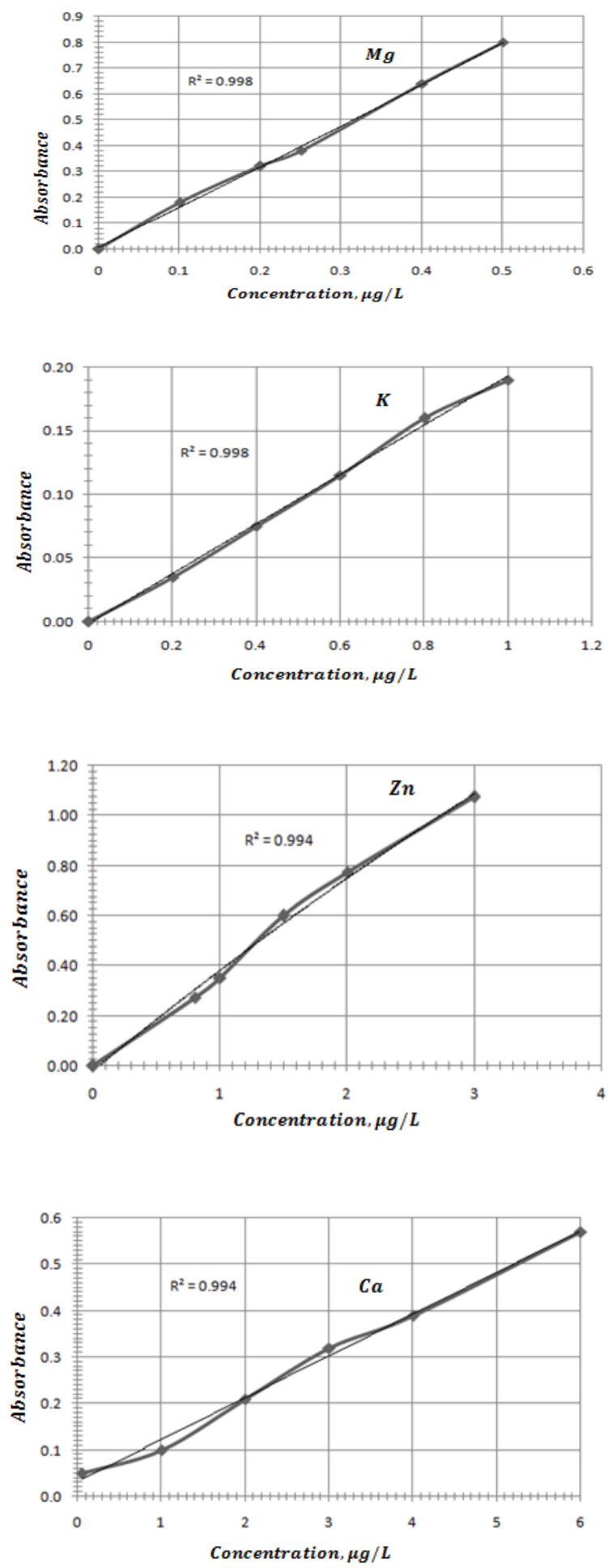
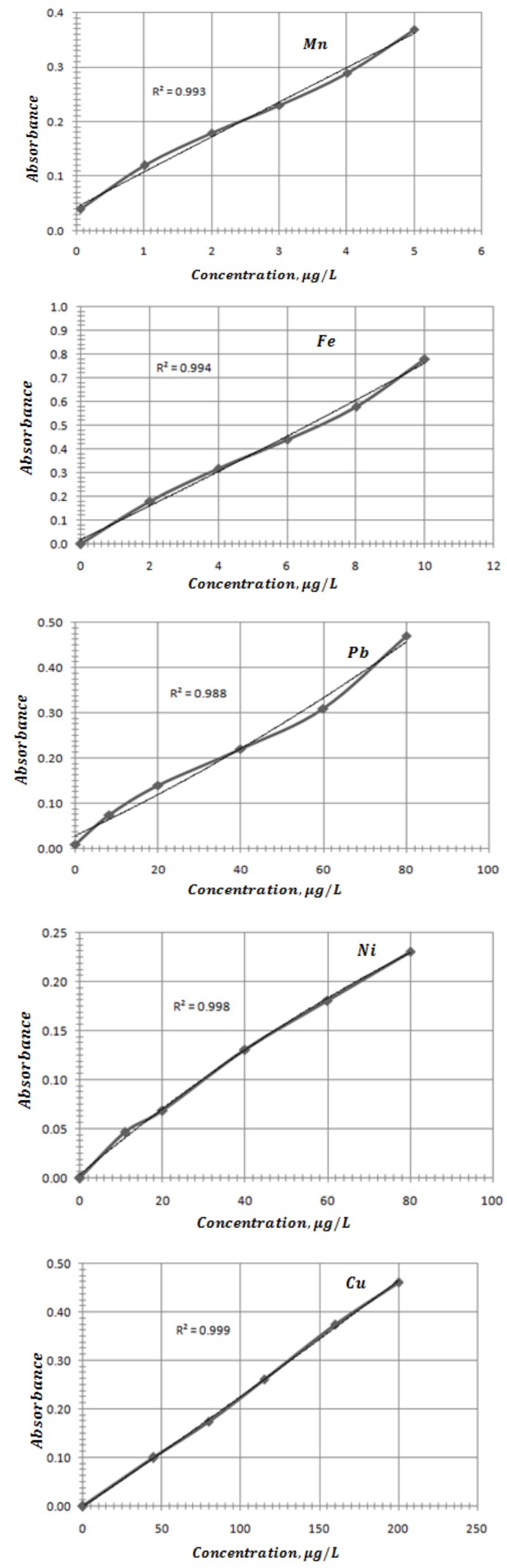

Fig. 2: Calibration curves of standards for $\mathrm{Mg}, \mathrm{K}, \mathrm{Zn}, \mathrm{Ca}, \mathrm{Mn}, \mathrm{Fe}, \mathrm{Pb}, \mathrm{Ni}$ and $\mathrm{Cu}$ 
Atomic spectroscopy is a commonly used method for the analysis of elements in free ions or elements in liable complexes. This method is used for all elements but gases, halogens, Sulphur and Phosphorous. This method measures accurate concentration of elements with throughput in the range of $10^{-10}$ to $10^{-13}$. Fig. (2) shows the calibration curves of various standard for magnesium, potassium, zinc, calcium, manganese, iron, lead, nickel, and copper. These calibration curves can be efficiently used to find the concentration of unknown, when the analyzing sample is prepared in the range of concentration shown in the graphs.

\section{DISCUSSION \& CONCLUSIONS}

The principle of atomic spectroscopy utilizes transitions between ground state and an upper excited state. This transition may be from ground state to higher excited state, or from ground state to any of the other excited states. The transition between ground state and the first excited state are highly sensitive and can be utilized for the analysis of samples with lower concentration. However, the excited atoms due to the transition from ground state to higher excited state are insensitive and are not analytically useful, because these excited atoms will have very small degree of absorption. For this reason, exciting energy in UV region spectrum can be used in metal and few metalloids, whereas for non-metals the energy must be taken in the vacuum UV region.

One may use quantum theory, as mentioned in the previous section, to both the type of atomic spectroscopy. In addition, the Grotrain diagram of an element help to deduce the absorption wavelengths by the atoms of the sample. The atomic absorption accounts its popularity in many laboratories for the advantages such as ease of operation, precision, relatively free from noises, samples are easy to prepare and rapidly handled using the atomizer.

Atomic absorption is common in many laboratories because of its detection limits, precision, ease of operation and relatively free from analytical interferences. In past, Atomic absorption spectroscopy made substantial impact on the procedures in the metallurgical laboratories. Refinements in equipment and techniques for using it should extend its usefulness in the period to come. As any other method, the atomic absorption spectroscopy limits its potential due to some drawbacks such as difficulty in handling solid samples and viscous liquid directly, difficulty in further dilution of a low analyte concentration, inefficient analytical result of thermally stable oxides. Thus, keeping in view of these advantages and disadvantages of atomic absorption spectroscopy it can be concluded that this method is the best suited for the determination of trace elements in large samples and advantageous in achieving a higher accuracy in determining the elements.

\section{REFERENCES}

[1] Alan Walsh, The application of atomic absorption spectra to chemical analysis. Spectrochim. Acta, 1955; 7:108.

[2] John Barry Dawson and William John Price, Alan Walsh - his influence on the development of atomic spectroscopy in the UK. Spectrochim. Acta Part B: Atomic Spectroscopy, 1999; 54(14): 20112015.
[3] Nancy Miller-lhli, Walter Slavin: scientist, mentor and friend. Spectrochim. Acta Part B: Atomic Spectroscopy, 2001; 56(9): 1501 1502.

[4] Bernhard Welz, Atomic absorption spectrometry - Pregnant after 45 years. Spectrochim. Acta Part B, 1999; 54: 2081-2094.

[5] Abdul Moiz Mohammed. UV-Visible spectrophotometric method and validation of organic compounds. European Journal of Engineering and Technology Research, 2018; 3(3): 8-11.

[6] Alan W. C. Menzies. A Study of atomic absorption spectroscopy. Analytical Chemistry, 1960; 32(8): 898-904.

[7] Jon C. Van Loon, Atomic absorption spectroscopy - selected methods, Academic Press, New York, 1980, pp. 4-9.

[8] Anthony F. Lagalante. Atomic absorption spectroscopy: A tutorial review. Applied Spectroscopy Reviews, 2007; 34(3): 173-189. 